\section{¿La imagen importa? Influencia de la vestimenta del traumatólogo en la elección y confianza del paciente}

\author{
STEFAN LOZIC ${ }^{1}$, PEDRO CHRISTIAN ARAVENA ${ }^{2, \mathrm{a}}$, \\ NICOLÁS MARTÍNEZ ${ }^{3}$, CATALINA PROBOSTE ${ }^{3, b}$, NICOLÁS PINTO ${ }^{3, b}$, \\ GABRIEL ALBORNOZ ${ }^{3, \mathrm{c}}$, PABLO LARRAGUIBEL ${ }^{1}$
}

\section{The impact of the orthopedic surgeons' attire on patient preferences}

Background: The formal appearance of health care professionals may influence their trustworthiness. Aim: To determine the effect of the orthopedic surgeon's attire on patients' perceptions of credibility and reliability of professionals. Material and Methods: In a cross-sectional design, 351 patients (mean age 43 \pm 17 years, $62 \%$ males) from the Department of Orthopedics and Traumatology of a Chilean regional hospital in southem of Chile were chosen to complete a questionnaire of attire preferences, in which five photographs with male and female orthopedic surgeons appeared (executive, formal attire, informal attire, scrubs and casual clothing). The influence of attire in the perception of physicians' trustworthiness to resolve medical situations was analyzed. Results: Forty four percent of patients had no physician gender predilection $(p=0.32$ ). Forty three percent of male and 38\% of female patients preferred the use of formal attire. In situations of credibility or confidence, all patients chose mostly the use of white coats with formal attire by professionals. The probability of choosing an orthopedic surgeon with a formal attire was significantly higher among patients who considered the attire and appearance of the professional to be very important (Odds ratio $=3.74 ; p<0.01)$. Conclusions: Patients prefer orthopedic surgeons wearing white coats and formal attire, which improves credibility of these professionals to correctly solve medical situations.

(Rev Med Chile 2017; 145: 987-995)

Key words: Clothing; Orthopedic Surgeons; Patient Preference; Physicians-Patient Relations.

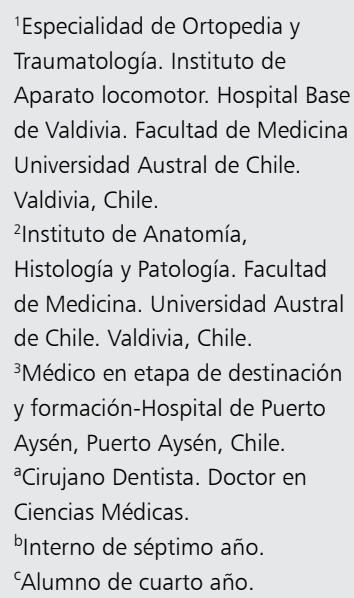

Financiamiento: Este trabajo fue financiado con recursos propios de los autores. No se recibió financiamiento externo para la realización de éste trabajo.

Conflicto de interés: Los autores declaran no tener conflictos de interés.

Recibido el 17 de marzo de 2017 aceptado el 8 de agosto de 2017

Correspondencia a:

Pedro Christian Aravena

Universidad Austral de Chile.

Facultad de Medicina

Campus Isla Teja S/N. Valdivia,

Chile.

paravena@uach.cl a relación médico-paciente es la base del éxito en la consulta médica, siendo la primera visita un momento clave para la buena impresión del médico tratante influenciado directamente por la limpieza, su vestimenta y aseo personal ${ }^{1,2}$. Por ello el rol del atuendo médico ha sido ampliamente debatido dado a la imagen pública que este representa. En general, los pacientes prefieren a aquel médico que usa delantal blanco y vestimenta formal la que favorese en la confianza y adherencia al tratamiento médico ${ }^{3-5}$.
No obstante, la elección ha variado dependiendo del tiempo, expectativas culturales y la generación de los pacientes ${ }^{3}$ incluyendo el tipo de especialidad médica $^{6,7}$

En un reciente estudio en Ortopedia y Traumatología, Jennings et al. ${ }^{8}$, demostraron que los pacientes tienen una leve preferencia hacia los médicos con delantal blanco. Sin embargo, no se presentan diferencias entre los distintos tipos de vestimenta en cuanto a la entrega de confianza y seguridad respecto al médico tratante; conside- 
rándose que la dignidad de la profesión médica no está ligada especificamente e su vestimenta, sino más bien al valor de su práctica profesional ${ }^{9}$. Asimismo, otros reportes afirman que la vestimenta del médico no influye respecto a la satisfacción en cuanto a su atención ${ }^{10,11}$. A la fecha, no existen estudios indexados en base electrónicas que pongan en evidencia la influencia del atuendo médico sobre la adherencia del paciente a los tratamientos prescritos en pacientes chilenos. Por ello, el propósito de este estudio es determinar la precepción de los pacientes con respecto a la vestimenta del médico traumatólogo en relación a la elección, credibilidad, adherencia y confianza de los pacientes tratados en un hospital del sur de Chile.

\section{Pacientes y Método}

Se diseñó un estudio de corte transversal basado en una encuesta adaptada al español ${ }^{3}$ usada en pacientes tratados en el departamento de Ortopedia y Traumatología del Hospital Regional de Valdivia en el mes de enero del año 2017. Tanto el estudio y el consentimiento informado fueron aprobados por el comité de ética de la Facultad de Medicina de la Universidad Austral de Chile.

\section{Selección de pacientes y tamaño de muestra}

Se incluyeron pacientes mayores de 18 años bajo tratamiento ambulatorio. Se excluyeron a pacientes con alteraciones cognitivas, visuales o motrices que le impidan comprender, leer y/o escribir adecuadamente.

Para el tamaño muestral se consideró las recomendaciones de Streiner \& Norman ${ }^{12}$ sugiriendo la participación de al menos diez observaciones por ítem del instrumento. Considerando que la encuesta original a usar consta de 25 ítems, se consideró un mínimo de 250 pacientes a encuestar.

\section{Adaptación de la encuesta al idioma español}

Para la adaptación del instrumento, se usó la metodología recomendada por Ramada-Rodilla et $\mathrm{al}^{13}$. Dos investigadores (PA, SL) con dominio del idioma inglés hicieron la traducción y adaptación al español del instrumento original ${ }^{3}$. Luego, un profesor de habla inglesa de la escuela de Idioma de la Universidad Austral de Chile realizó la traducción inversa del texto el cual fue comparado con el instrumento original detectando solo dos frases con diferencias gramaticales.
El borrador de la escala fue revisado por cinco personas del Departamento de Ortopedia y Traumatología sugiriendo los siguientes cambios: en la elección del sexo del médico agregar la opción "me es indiferente", una nueva opción del médico usando un delantal blanco abierto con ropa semiformal y una última pregunta denominada $E n$ general, ¿a qué traumatólogo elegiría?. Para evaluar su validez de criterio, un investigador (SL) entregó el borrador impreso a 10 pacientes voluntarios en la sala de espera del servicio, sugiriendo aumentar el tamaño de letra a una tipografía Arial número 12 y especificar la elección de sólo una alternativa. Para comprobar la fiabilidad intra-observador, se les entregó el mismo borrador a los mismos pacientes 15 días posteriores, calculando una correlación de Pearson promedio rho $=0,89$ en las preguntas analizadas.

Finalmente, la encuesta constó con dos segmentos:

1. Registro de datos sociodemográficos, elección de importancia de la apariencia y vestimenta y del sexo del traumatólogo.

2. Elección de cinco tipos de vestimenta según sexo definidos como:

a. Ejecutivo (traje con corbata o falda, sin delantal blanco).

b. Uso delantal blanco formal (camisa y corbata o blusa y falda, con delantal blanco cerrado).

c. Uso delantal blanco informal (camisa abierta sin corbata o camiseta y falda, ambos con delantal blanco abierto).

d. Traje clínico.

e. Ropa casual (jeans, polera y zapatillas para ambos sexos) (Figura 1a y 1b).

Para ello, este segmento contó con tres dominios, cada una con cuatro escenarios o ítems: preferencia ante una situación médica, preferencia según entrega de confianza en su diagnóstico o tratamiento y preferencia de acuerdo a entrega de valores inherentes del profesional.

\section{Uso de la encuesta}

Se aplicó la encuesta a pacientes en sala de espera de la consulta ambulatoria según los criterios de selección ya mencionados. Luego de explicar verbalmente de que trataba el estudio y la aprobación del consentimiento informado, los investigadores (CP, GA, NP) entregaron la en- 

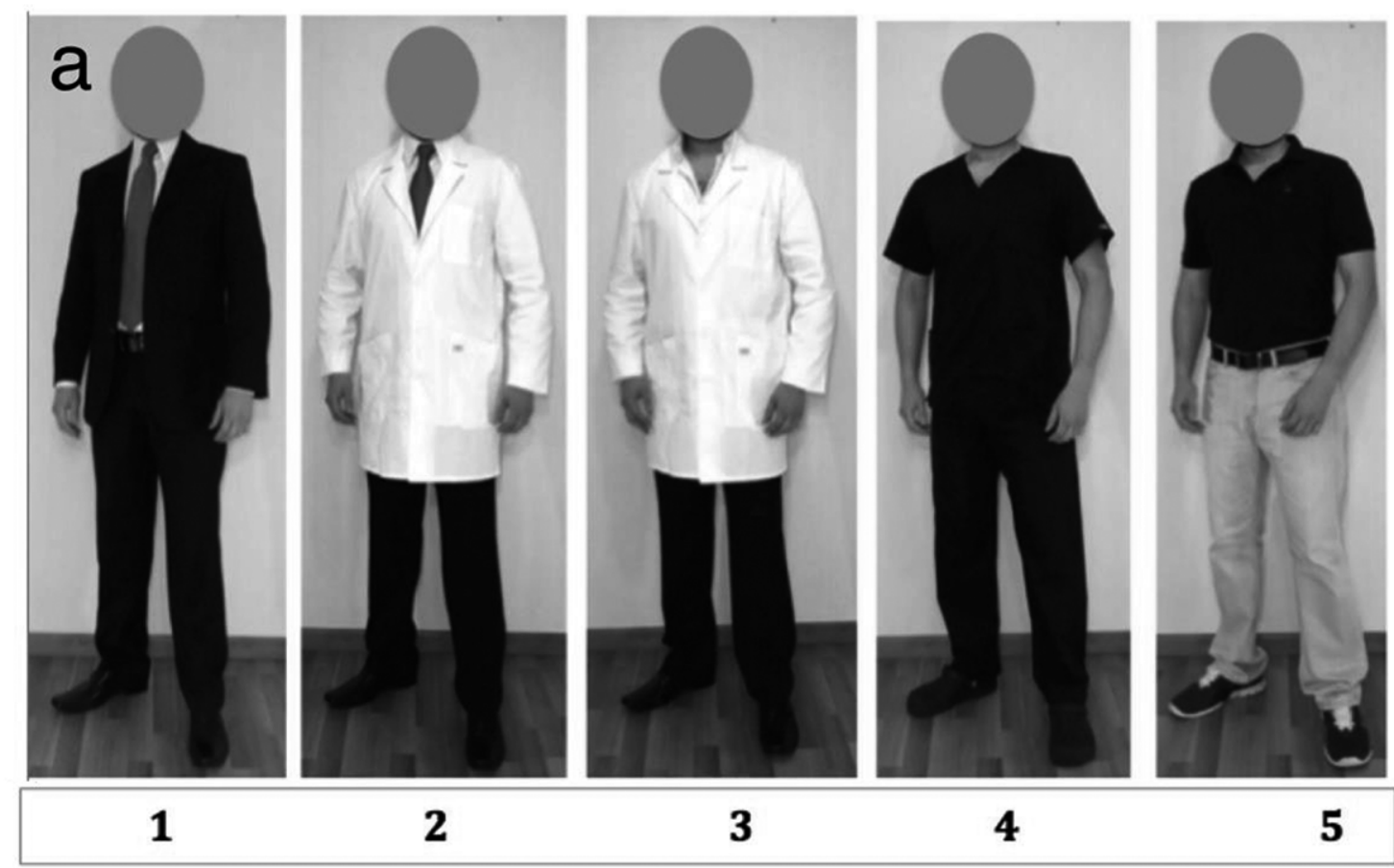

2

3

4

5
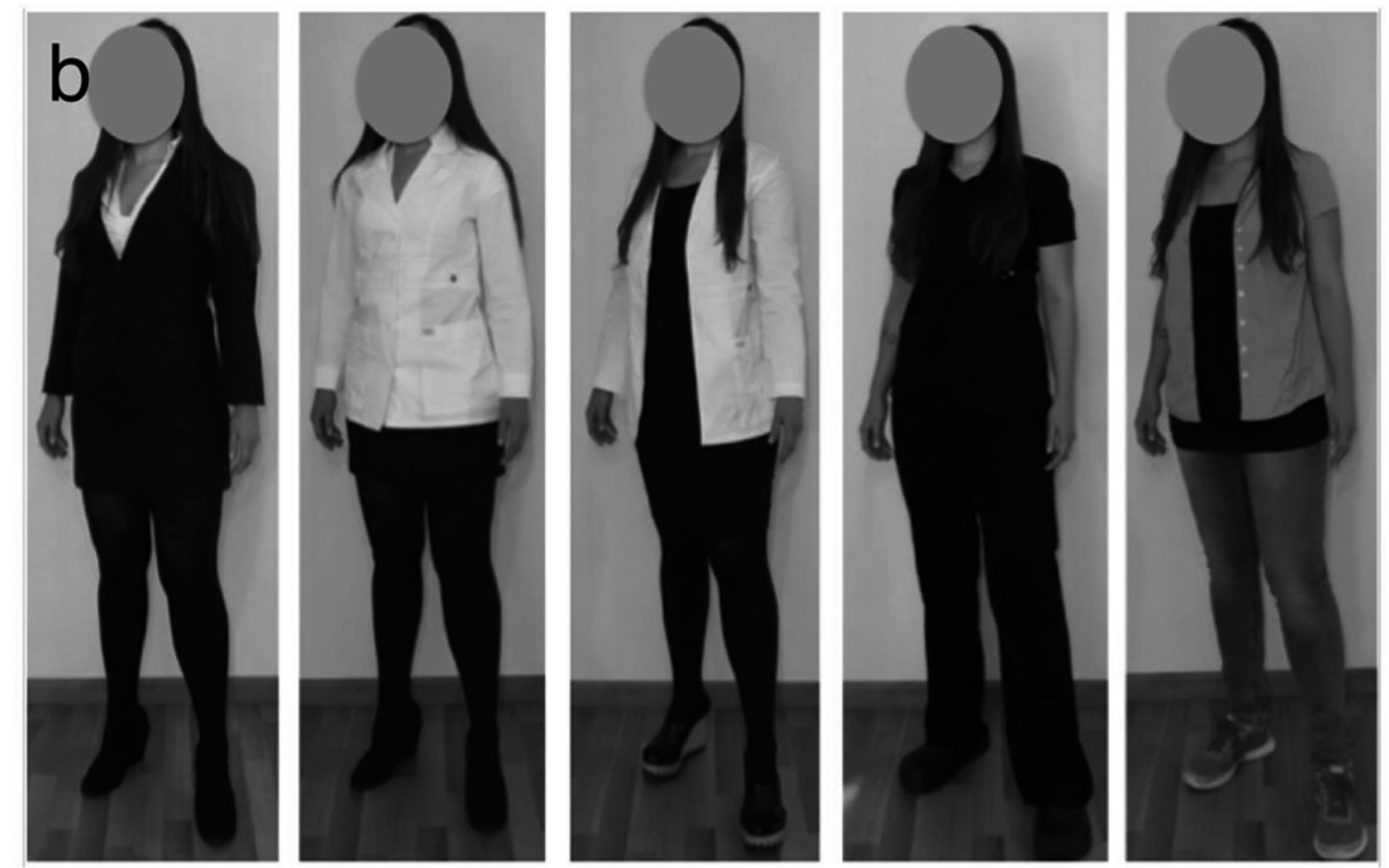

1

2

3

4

5

Figura 1. Modelo médico hombre (a) y mujer (b) con los 5 opciones de vestimenta. 
cuesta y un lápiz de tinta para ser completada sin límite de tiempo. Para evitar el sesgo de selección, todos los investigadores vestían con ropa casual, no coincidiendo con ninguna imagen presente en la encuesta.

\section{Análisis de datos}

Los resultados fueron recopilados y tabulados por un investigador (NP) en una planilla de datos electrónica (Google Drive. Google Inc. CA. USA). Por cada paciente se registró edad, sexo, región de procedencia, nivel de estudios y tipo de seguro de salud. Un tercer investigador (PA) realizó el análisis estadístico a dos colas. Se comparó la influencia del sexo y la edad del paciente en la importancia del vestir y la elección del sexo del médico traumatólogo $\left(\chi^{2}\right.$, t-test; $\left.\mathrm{p}<0,05\right)$. Se realizó un análisis descriptivo de la frecuencia de pacientes y el tipo de vestimenta elegida por cada pregunta o situación presentando esto resultados en porcentajes y gráfico de barras apiladas. Se creó un modelo de regresión logística multivariante para determinar las características significativamente asociadas a la mayor preferencia de la vestimenta y sexo del médico traumatólogo. Estos resultados se resumieron usando Odds ratios e intervalos de confianza a 95\% (STATA v.14.0. STATA Corp. Texas, USA).

\section{Resultados}

Participaron en total 351 pacientes con un promedio de edad de 43,5 \pm 17,2 años (min: 18 Max: $90)$ de los cuales 217 (61,8\%) fueron hombres. El detalle se presenta en la Tabla 1.

Considerando la importancia del vestir de los traumatólogos, 67,3\% de los pacientes la consideraron "muy importante". Respecto a la elección del sexo, 43,8\% de los encuestados indicaron como "indiferente". No se presentaron diferencias estadísticamente significativas en las opciones de respuesta según el sexo de los participantes (Tabla 2).

Respecto a las opciones de los traumatólogos hombres, en la pregunta ¿A qué traumatólogo hombre prefiere para las situaciones...? en el ítem "problema médico menor" 35,3\% de los pacientes optaron por el uso de traje clínico (opción No 4). Por otra parte, en los ítems "problema médico mayor", "someterse a cirugía ortopédica sin urgencia" y "emergencia traumatológica con riesgo de vida" $36,8 \%, 36,8 \%$ y $38,5 \%$ de los pacientes optaron por el uso de delantal formal (opción No 2), respectivamente.

En la pregunta ¿a cuál de estos traumatólogos hombre...? destaca la elección del uso de delantal formal para el ítem "entrega más confianza en el

Tabla 1. Datos sociodemográfico de los participantes del estudio según sexo

\begin{tabular}{|llccc|}
\hline Variable & Ítem & Hombre n: 134 & Mujer n: 217 & Total n: $\mathbf{3 5 1}$ \\
Edad & Promedio \pm DE & $41,8 \pm 16,5$ & $44,5 \pm 17,6$ & $43,5 \pm 17,2$ \\
Región procedencia & RM & 2 & 1 & 3 \\
& VII & 0 & 1 & 1 \\
& VIII & 2 & 0 & 1 \\
& IX & 1 & 0 & 342 \\
NIV & 128 & 214 & 2 \\
Nivel educación & X & 1 & 1 & 104 \\
& Básico & 40 & 64 & 142 \\
Tipo de seguro de salud & Medio & 59 & 83 & 55 \\
& Técnico & 16 & 39 & 512 \\
& Superior & 19 & 31 & 20 \\
\hline
\end{tabular}


Vestimenta del médico en la elección y confianza del paciente - S. Lozic et al

Tabla 2. Porcentaje de participantes según la pregunta de la importancia de la vestidura y el sexo del traumatólogo (n: 351)

\begin{tabular}{|c|c|c|c|c|c|}
\hline Variable & Ítem & $\begin{array}{c}\text { Total } \\
(n=351)\end{array}$ & $\begin{array}{c}\text { Hombre } \\
(n=217)\end{array}$ & $\begin{array}{c}\text { Mujer } \\
(n=134)\end{array}$ & p* \\
\hline \multirow[t]{2}{*}{ ¿Qué importancia le da a la apariencia y al vestir? } & Muy importante & 67,3 & 64,9 & 70,9 & \multirow[t]{2}{*}{0,25} \\
\hline & No importa & 32,7 & 35,1 & 29,1 & \\
\hline \multirow[t]{3}{*}{ Para un problema traumatológico, usted prefiere: } & Hombre & 36,8 & 32,2 & 41,3 & \multirow[t]{3}{*}{0,32} \\
\hline & Mujer & 19,4 & 19,6 & 19,4 & \\
\hline & Me es indiferente & 43,8 & 48,2 & 39,3 & \\
\hline
\end{tabular}

${ }^{*} \chi^{2}(p<0,05)$.

diagnóstico y tratamiento" (48,4\%). En la pregunta ¿Cuál de estos traumatólogos hombre les parece...? Todos los pacientes optaron mayoritariamente por el uso de delantal formal, destacando la elección de las opciones con "más conocimiento y competente" (53\%), "más responsable" (49,9\%) y "más autoridad y control" (47\%).

Finalmente, en la pregunta En general, ¿a qué traumatólogo elegiría? 42,7\% de los pacientes optaron por el uso de delantal formal, seguido en $23,3 \%$ por el uso de delantal informal (opción No 3). El detalle de la elección del vestir para el traumatólogo hombre según la pregunta y sus opciones se detalla en la Figura 2.
Respecto a las opciones de las traumatólogos mujeres, la pregunta ¿A qué traumatólogo mujer prefiere para las siguientes situaciones...? en el ítem "problema médico menor" 33,3\% de los pacientes optaron el uso de traje clínico. Por otra parte, en los ítems "problema médico mayor", "someterse a cirugía ortopédica sin urgencia" y "emergencia traumatológica riesgo vida" 33,3\%, 34,8\% y $36,8 \%$ optaron por el uso de delantal formal, respectivamente.

En la pregunta ¿a cuál de estos traumatólogos mujer...? destaca la elección del delantal formal en el ítem "haría más caso" $(48,4 \%)$ seguido por "volvería atenderse con él o lo recomienda" $(39,6 \%)$.

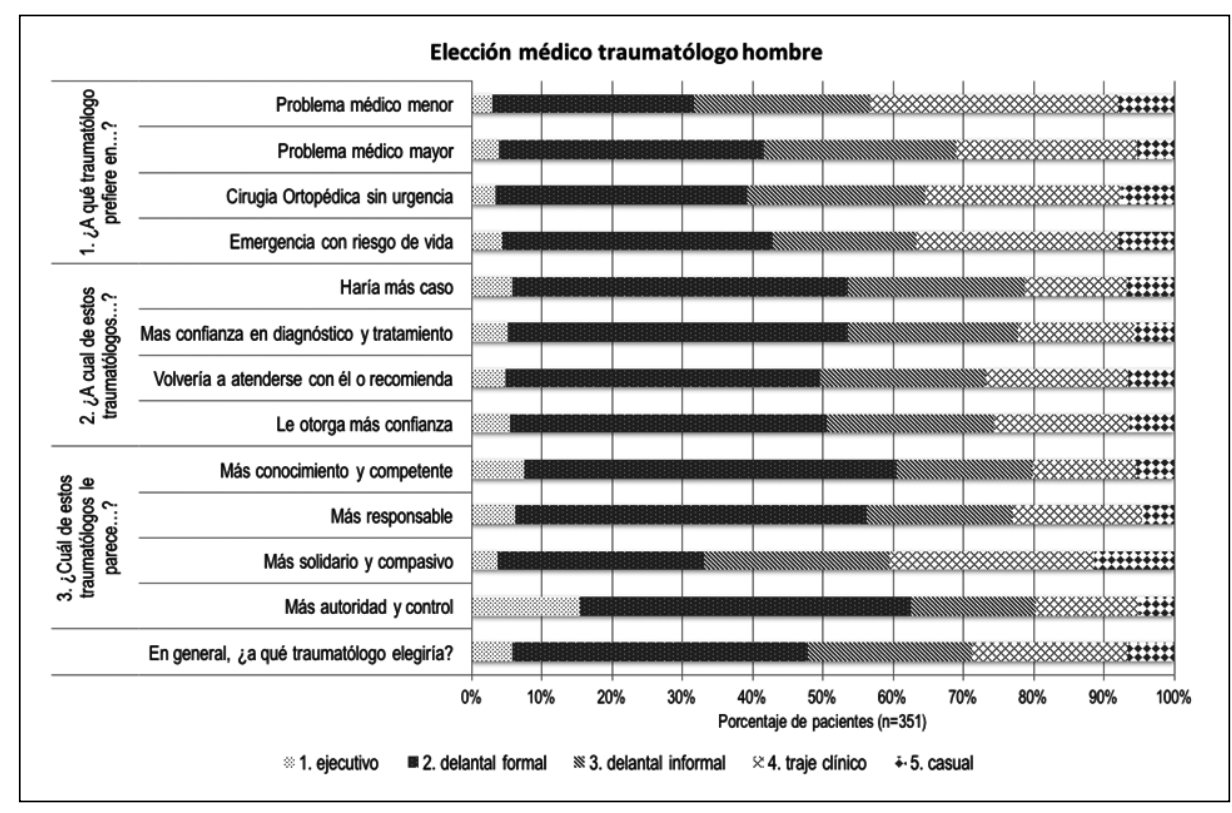

Figura 2. Distribución de participantes en la elección de la vestimenta de traumatólogos acorde a las preguntas de la encuesta $(n=351)$. 
Por otra parte, en los ítems de la pregunta ¿Cuál de estos Traumatólogos mujer les parece...? Todas ellas optaron mayoritariamente por el uso de delantal formal. Finalmente, en la pregunta En general, ¿a qué traumatólogo elegiría?, 37,6\% de los pacientes optaron por el uso de delantal formal, seguido en $28,2 \%$ para el traje clínico. El detalle de la elección del vestir para el traumatólogo mujer según la pregunta y sus opciones se detalla en la Figura 3.
La distribución de la edad de los pacientes no presentó una tendencia en la elección de la vestimenta. Sin embargo, se presentaron diferencias significativas en las edades de los encuestados para la elección del uso de delantal informal $(\mathrm{p}=0,0003)$ y el uso de ropa casual $(\mathrm{p}=0,03)$ (Figura 4).

Considerando que en general el uso de delantal formal fue la más escogida por los pacientes para

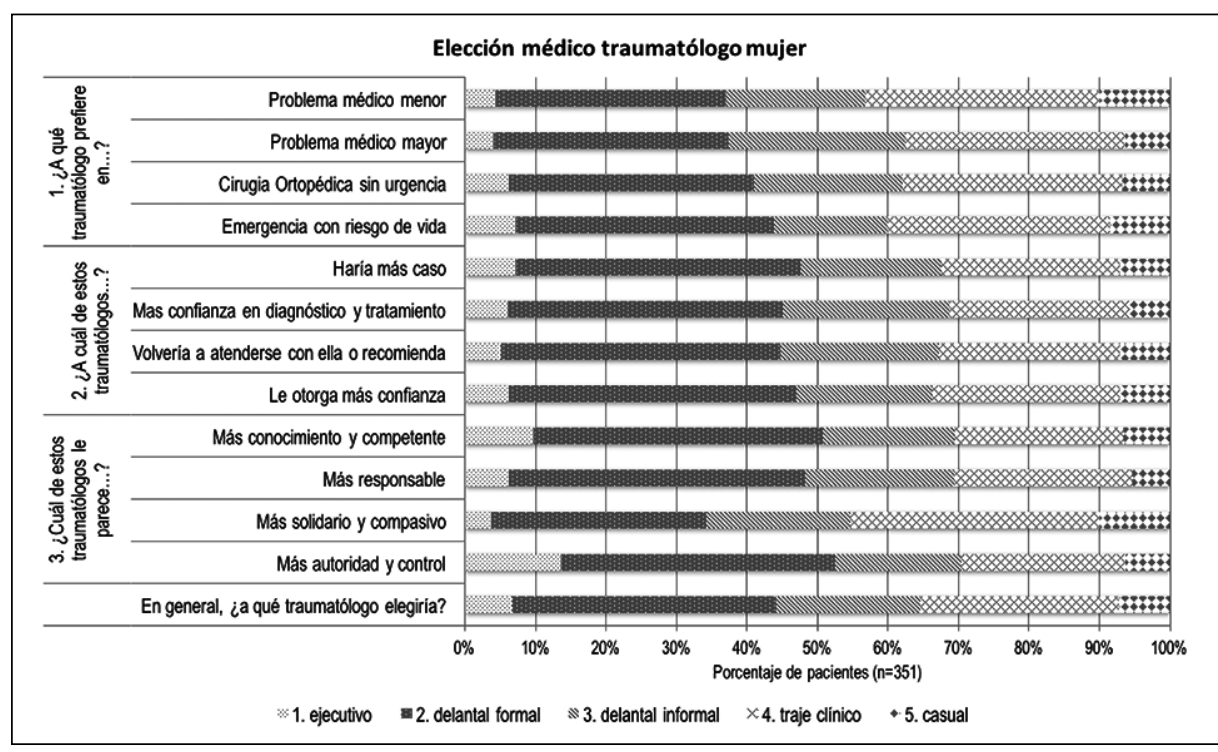

Figura 3. Distribución de participantes en la elección de traumatólogas acorde a las preguntas de la encuesta $(n=$ 351).

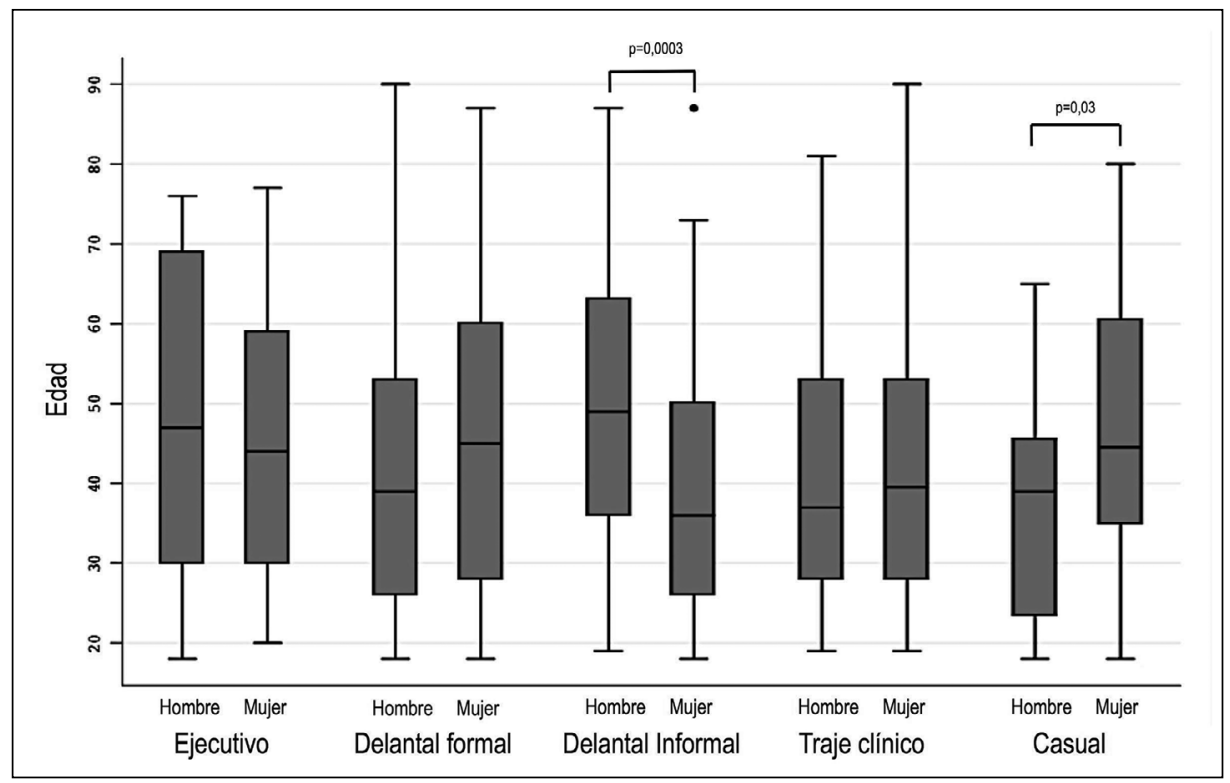

Figura 4. Distribución de las edades de los pacientes según el sexo del traumatólogo por cada opción de vestimenta a elegir (t-test; $\mathrm{p}<0,05$ ). 
Tabla 3. Análisis de regresión logística como modelo predictivo en la elección general del uso de delantal formal (opción $\mathrm{N}^{\circ} 2$ ) según las variables de los pacientes

\begin{tabular}{|c|c|c|c|c|c|c|}
\hline \multirow[b]{2}{*}{ Variable } & \multicolumn{3}{|c|}{ Preferencia traumatólogo hombre } & \multicolumn{3}{|c|}{ Preferencia traumatológo mujer } \\
\hline & $\begin{array}{l}\text { Odds } \\
\text { ratio }\end{array}$ & $\begin{array}{l}95 \% \text { int. } \\
\text { confianza }\end{array}$ & $\begin{array}{c}\text { Valor } \\
\mathbf{p}\end{array}$ & $\begin{array}{l}\text { Odds } \\
\text { ratio }\end{array}$ & $\begin{array}{l}95 \% \text { int. } \\
\text { confianza }\end{array}$ & $\begin{array}{c}\text { Valor } \\
\mathbf{p}\end{array}$ \\
\hline Sexo & 0,76 & $0,48-1,2$ & 0,24 & 1,43 & $0,9-2,27$ & 0,12 \\
\hline Edad & 0,98 & $0,97-0,99$ & 0,05 & 1,01 & $0,99-1,02$ & 0,21 \\
\hline Tipo seguro de salud & 0,93 & $0,61-1,44$ & 0,76 & 0,7 & $0,43-1,14$ & 0,16 \\
\hline Nivel de educación & 0,84 & $0,49-1,43$ & 0,52 & 0,71 & $0,41-1,21$ & 0,21 \\
\hline Importancia del vestir & 3,74 & $1,03-4,77$ & $<0,0001$ & 3,48 & $0,86-4,99$ & $<0,0001$ \\
\hline
\end{tabular}

traumatólogos de ambos sexos (Figuras 2 y 3 ), el modelo predictivo de la regresión logística no demostró asociación significativa con características de los pacientes. Sin embargo, la probabilidad de escoger al traumatólogo con delantal formal fue significativamente mayor en los encuestados que consideraron como muy importante la vestimenta y la apariencia del profesional $(\mathrm{p}<0,0001)(\mathrm{Ta}-$ bla 3$)$.

\section{Discusión}

El uso delantal blanco con una ropa formal (opción $\mathrm{N}^{\circ}$ 2) es la opción más elegida por los encuestados para traumatólogos de ambos sexos. La probabilidad de escoger al traumatólogo con delantal formal fue significativamente mayor en los encuestados que consideraron como muy importante la vestimenta y la apariencia del profesional percibido como un médico con mejores competencias clínicas y actitudinales, al que harían más caso en las indicaciones, otorga más confianza y con quien acudirían a atenderse nuevamente.

El delantal blanco ha sido símbolo de la profesión médica desde que fue adoptado en la labor en laboratorio en la mitad del siglo XIX y ha sido admitido como un símbolo de la profesión médica ${ }^{14}$. Sin embargo, en las últimas décadas ha variado a combinaciones de trajes semi formales y uso de ropa clínica de colores otorgando un aspecto más modesto a la vestimenta ${ }^{15}$. Por otra parte, últimamente se ha intensificado los debates sobre su uso considerando la posibilidad de contaminación cruzada y la potencial fuente de infecciones adquiridas en los centros clínicos ${ }^{16}$.
Es así como países como Reino Unido y Estados Unidos han adoptado la política de tener desnudo el antebrazo bajo el codo ("bare below the elbow") con el propósito de minimizar la transmisión de infecciones nosocomiales particularmente en unidades de cuidados intensivos y críticos. Sin embargo, existen reportes ${ }^{9}$ que rechazan esta postura, considerando que no existe evidencia suficiente para concluir la presencia de infección cruzada, fundamentando que la dignidad de la profesión médica no está ligada a una forma específica de vestimenta, sino más bien al valor de la práctica médica. A pesar de estos discusiones, reportes en el cual los pacientes fueron advertidos sobre el riesgo de infección cruzada siguieron eligiendo a los profesionales con delantal blanco ${ }^{17}$.

La elección del uso de delantal blanco y vestimenta formal puede deberse a la percepción de limpieza y en su rol como símbolo médico que lo diferencia de otros funcionarios ${ }^{3,4,14,18}$. Sin embargo, otros reportes demuestran que los pacientes confían en sus médicos independiente de la forma en que este se vista, incluso si ésta no les gusta ${ }^{10,11}$. Estas diferencias podrían estar relacionadas tanto como factores socioculturales de los pacientes, la edad y el tipo de especialidad médica. Considerando que el estudio fue realizado en el servicio de Ortopedia y Traumatología de un hospital público de una región del sur de Chile, las condiciones sociodemográficas no demostraron ser un factor que influyera en la elección del vestir del médico traumatólogo (Tabla 3, Figura 4). Otros reportes ${ }^{3,19-21}$ describen que los pacientes de edad avanzada tienen una predilección del médico con uso de delantal blanco posiblemente por el hecho de que los pacientes mayores son generalmente 
más conservadores, percibiendo con ello un mayor nivel de experticia y capacitación en comparación con el uso de teñida no formal.

Por otra parte, se ha demostrado que la ubicación geográfica también presenta una relación en la elección de la vestimenta. Países como Canadá, Reino Unido, Escocia, Irlanda, Japón y Korea del Sur el uso de delantal blanco formal influye en la mejor percepción y confianza del médico tratante. Por otra parte, 4 de 8 estudios estadounidenses no demostraron mayor preferencia en el tipo de vestimenta $^{22}$. En Sudamérica, un reporte argentino ${ }^{23}$ demostró que tanto acompañantes y niños mayores de seis años preferían la atención de un médico usando delantal blanco, incluso $88 \%$ de los niños rechazaría la atención de un médico pediatra que no lo usara. Un estudio brasileño ${ }^{24}$ demostró también una elección prioritaria de uso de ropa blanca (polera y pantalón) condicionado por las altas temperaturas que los médicos se ven expuestos en mayor parte del año.

La especialidad médica a analizar y el contexto de la atención del paciente condicionan la elección de la vestimenta del médico tratante ${ }^{22}$. Nuestro reporte en Ortopedia y Traumatología favorece a la elección del médico del tratante con delantal blanco y uso de corbata de manera formal, similares a otros reportes de la especialidad ${ }^{9}$. Por otra parte estudios en pediatría ${ }^{6}$, psiquiatría $^{7}$ y ginecología $a^{25}$ no demuestra una preferencia de la forma de vestir del profesional, siendo incluso un aspecto que distancia la confianza y cercanía que se requiera con el médico tratante.

En nuestro estudio consideramos algunas limitaciones. Primero, el uso de una encuesta adaptada al español podría tener variaciones en la medición del constructo para la que fue hecha originalmente. Segundo, su uso en la sala de espera podría generar el sesgo de respuesta, considerando el tránsito de los médicos en el lugar o la influencia de la vestimenta del médico tratante. Por último, las respuestas de los encuestados están supeditadas solo a la fotografía de la vestimenta del médico desconocido, sin considerar elementos determinantes respecto a la preferencia tales como el lenguaje verbal y no verbal, aseo personal, comportamiento, el carisma, la empatía, el tono y el volumen de la voz, entre otros ${ }^{1,3}$. A pesar de estas limitaciones, nuestro estudio es el primer reporte que analiza en Chile la importancia de la vestimenta del médico en Traumatología asociado a la elección, credibilidad y confianza de los pacientes, usando una metodología fehaciente en la adaptación al español del instrumento original y analizando la asociación de dicha elección con características sociodemográficas de los participantes.

En conclusión, los pacientes del Servicio de Ortopedia y Traumatología de un hospital al sur de Chile prefieren a médicos con vestuario formal y uso de delantal blanco. Estos resultados sugieren a la comunidad médica usar traje formal con delantal blanco durante la atención de pacientes ya que podría influir favorablemente en la percepción, su confianza y eventualmente en la adherencia y retorno a la atención médica. Se sugiere nuevos estudios similares en distintos centros hospitalarios y especialidades médicas en nuestro país.

\section{Referencias}

1. Short D. First impressions. Br J Hosp Med 1993; 50 (5): 270-1.

2. Goffman E. La Presentación de la persona en la vida cotidiana. H.F. Martínez de Murguía; 1987. p. 273.

3. Rehman SU, Nietert PJ, Cope DW, Kilpatrick AO. What to wear today? Effect of doctor'sattire on the trust and confidence of patients. Am J Med 2005; 118 (11): 127986.

4. Gherardi G, Cameron J, West A, Crossley M. Are we dressed to impress? A descriptive survey assessing patients' preference of doctors' attire in the hospital setting. Clin Med Lond Eng 2009; 9 (6): 519-24.

5. Kurihara H, Maeno T, Maeno T. Importance of physicians' attire: factors influencing the impression it makes on patients, a cross-sectional study. Asia Pac Fam Med 2014; 13 (1): 2.

6. Hofmann J, Zotter H, Kerbl R. How to dress as a paediatrician? Acta Paediatr 2012; 101 (12): 1260-4.

7. Nihalani ND, Kunwar A, Staller J, Lamberti JS. How should psychiatrists dress?- a survey. Community Ment Health J 2006; 42 (3): 291-302.

8. Jennings JD. Ciaravino SG, Ramsey FV, Haydel C. Physicians' attire influences patients' perceptions in the urban out patient orthopaedic surgery setting. Clinical Orthopaedics and Related Research 2016; 474 (9): 1908-18. Disponible en: http://link.springer.com/ article/10.1007/s11999-016-4855-7 [consultado el 2 de marzo de 2017].

9. Palacios-González C, Lawrence DR. Substance over style: is there something wrong with abandoning the white coat? J Med Ethics 2015; 41 (6): 433-6. 
10. Edwards RD, Saladyga AT, Schriver JP, Davis KG. Patient attitudes to surgeons' attire in anout patient clinic setting: substance overstyle. Am J Surg 2012; 204 (5): 663-5.

11. Toquero L, Aboumarzouk O, Owers C, Chiang R, Thiagarajah S, Amin S. Bare below the elbows-The Patient's Perspective. Webmed Central Quality and patient safety 2011;2(4):WMC001401. Disponible en: https://www. webmedcentral.com/article_view/140 [consultado el 3 de marzo de 2017].

12. Streiner DL, Norman GR, Cairney J. Health Measurement Scales: A Practical Guide to Their Development and Use. Fifth Edition. Oxford University Press; 2015. p. 54.

13. Ramada-Rodilla JM, Serra-Pujadas C, Delclós-Clanchet GL. Adaptación cultural y validación de cuestionarios de salud: revisión y recomendaciones metodológicas. Salud Pública México 2013; 55 (1): 57-66.

14. Csendes JA, Korn BO. ¿Qué representa el delantal blanco? Rev Chil Cir 2008; 60 (6): 567-9.

15. Hardy S, Corones A. Dressed to Heal: The Changing Semiotics of Surgical Dress. FashTheory 2016; 20 (1): 27-49.

16. Treakle AM, Thom KA, Furuno JP, Strauss SM, Harris $\mathrm{AD}$, Perencevich EN. Bacterial contamination of health care workers' whitecoats. Am J Infect Control 2009; 37 (2): 101-5.

17. So EC, Fung FH, Yeung JK, Chow LH, Kwok JS, Lam $\mathrm{RL}$, et al. Patient perception of physician attire before and after disclosure of the risks of microbial contamination. Int J Med Stud 2013; 1 (3): 109-14.

18. Gooden BR, Smith MJ, Tattersall SJ, Stockler MR. Hos- pitalised patients' view son doctors and whitecoats. Med J Aust 2001; 175 (4): 219-22.

19. Sebo P, Herrmann FR, Haller DM. White coat in primary care: what do patients think today? Cross-sectional study. Swiss Med Wkly 2014; 144: w14072.

20. Lill MM, Wilkinson TJ. Judging a book by its cover: descriptive survey of patients' preferences for doctors' appearance and mode of address. BMJ 2005; 331 (7531): 1524-7.

21. Sotgiu G, Nieddu P, Mameli L, Sorrentino E, Pirina P, Porcu A, et al. Evidence for preferences of Italian patients for physician attire. Patient Prefer Adherence 2012; 6: 361-7.

22. Petrilli CM, Mack M, Petrilli JJ, Hickner A, Saint S, Chopra V. Understanding the role of physician attire on patient perceptions: a systematic review of the literature-targeting attire to improve likelihood of rapport to improve likelihood of rapport (TAILOR) investigators. BMJ Open 2015; 5 (1): e006578.

23. Eymann AM, Maxit C, Cacchiarelli N, Imach E, Wahren C. La vestimenta del médico pediatra en el consultorio hospitalario. Arch Argent Pediatría 2005; 103 (3): 212-7.

24. Yonekura CL, Certain L, Karen SKK, Alcântara GAS, Ribeiro LG, Rodrigues-Júnior AL, et al. Impressões de pacientes, médicos e estudantes de medicina quanto a aparência dos médicos. Rev Assoc Médica Bras 2013; 59 (5): 452-9.

25. Fischer RL, Hansen CE, Hunter RL, Veloski JJ. Does physician attire influence patient satisfaction in an out patient obstetrics and gynecology setting? Am J Obstet Gynecol 2007; 196 (2): 186.e1-5. 